\title{
Modelling the nova rate in galaxies
}

\author{
F. Matteucci ${ }^{1}$, A. Renda ${ }^{1}$, A. Pipino ${ }^{1}$, and M. Della Valle ${ }^{2}$ \\ 1 Dipartimento di Astronomia, Universitá di Trieste, via G.B. Tiepolo 11, 34131 Trieste, Italy \\ 2 Osservatorio Astronomico di Arcetri, Largo E. Fermi, Firenze, Italy
}

Received 10 February 2003 / Accepted 3 April 2003

\begin{abstract}
We compute theoretical nova rates as well as type Ia SN rates in galaxies of different morphological type (Milky Way, ellipticals and irregulars) by means of detailed chemical evolution models, and compare them with the most recent observations. The main difference among the different galaxies is the assumed history of star formation. In particular, we predict that the nova rates in giant ellipticals such as M 87 are $\sim 100-300$ nova $\mathrm{yr}^{-1}$, about a factor of ten larger than in our Galaxy $\left(\sim 25\right.$ nova yr$\left.^{-1}\right)$, in agreement with very recent estimates from HST data. The best agreement with the observed rates is obtained if the recurrence time of novae in ellipticals is assumed to be longer than in the Milky Way. This result indicates that the star formation rate in ellipticals, and in particular in M 87, must have been very efficient at early cosmic epochs. We predict a nova rate for the LMC of 1.7 nova $\mathrm{yr}^{-1}$, again in agreement with observations. We compute also the $K$ - and $B$-band luminosities for ellipticals of different luminous mass and conclude that there is not a clear trend for the luminosity specific nova rate with luminosity among these galaxies. However, firm conclusions about ellipticals cannot be drawn because of possible observational biases in observing these objects. The comparison between the specific nova rates in the Milky Way and the LMC indicates a trend of increasing nova rate passing from the Galaxy towards late-type spirals and Magellanic irregulars.
\end{abstract}

Key words. stars: novae, cataclysmic variables - stars: supernovae: general

\section{Introduction}

Classical novae are binary systems, where one component is a white dwarf (either carbon-oxygen or neon-oxygenmagnesium), accreting hydrogen rich material from a less evolved companion. When the pressure and temperature at the bottom of the accreted layer exceed critical values (e.g. Livio 1992) the nuclear reactions are ignited and novae exhibit a sudden and rapid increase of their brightness. The thermonuclear runaways are responsible for the production of interesting chemical species, such as ${ }^{13} \mathrm{C},{ }^{15} \mathrm{~N},{ }^{17} \mathrm{O},{ }^{22} \mathrm{Na},{ }^{26} \mathrm{Al},{ }^{22} \mathrm{Ne}$ and possibly ${ }^{7} \mathrm{Li}$ (see Jose \& Hernanz 1998; Gehrz et al. 1998; Della Valle et al. 2002) and for ejection of the accreted envelope. Although the matter processed through nova explosions is a small fraction (a few $10^{-3}$ ) of the total mass of the interstellar gas and dust in the Milky Way, the concentrations of these rare isotopes in the nova ejecta can be enhanced, relative to solar abundances, by factors $10^{2-3}$, therefore making novae non-negligible contributors to the galactic nucleosynthesis (Romano et al. 1999, 2001; Romano \& Matteucci 2003). Novae have been observed not only in our Galaxy but also in the Local Group (e.g. Hubble 1929, Arp 1956 and Rosino 1964) and beyond, in Virgo (Pritchet \& van den Bergh 1987) in M 81 (Shara et al. 1999) and in Fornax (Della Valle \& Gilmozzi 2002). From these studies some estimates of nova rates have been derived

Send offprint requests to: F. Matteucci, e-mail: matteucci@ts . astro.it and they are shown in Table 1, where the Hubble type of the galaxies is indicated in Col. 1, the nova rate in $\mathrm{yr}^{-1}$ in Col. 2, the $K$-band luminosity of the object in Col. 3 and in Col. 4 the luminosity specific nova rate in units of $\mathrm{yr}^{-1} 10^{-10} L_{\odot, K}\left(L S N R_{K}\right)$. These rates are taken from Della Valle (2002) and Shafter et al. (2000).

In this paper we use the chemical evolution models elaborated by Chiappini et al. (2001), Matteucci et al. (1998) and Calura et al. (2003) for the Milky Way, Ellipticals and late Spirals, respectively, to compute the nova rate in "disk" dominated and "bulge" dominated systems with the intent of checking whether the different observed nova rates can be reproduced under the assumption that the main difference between galactic morphological types should be ascribed to the different histories of star formation. Moreover, our study is aimed at deriving important constraints on some key parameters of nova theory, which cannot be directly inferred from observations, such as the recurrence time between the outbursts, the fraction of binary systems which give rise to nova systems, the delay time with which the first novae appear relative to the beginning of star formation. The use of such models is appropriate since they can reproduce the basic parameters characterizing the respective parent galaxies such as the rate of type Ia SNe, $B$ and $K$ luminosities of the parent galaxies, the $[\alpha / \mathrm{Fe}]$ ratios and global metallicities in the stellar populations and gas. Finally, we note that a fraction of type Ia SNe can originate from WDs in $\mathrm{CV}$-like systems, after reaching the Chandrasekhar mass limit 
Table 1. Extragalactic nova rates and luminosity-specific nova rates from Della Valle (2002). The latest estimate for M 87(M 87*) is from Shara \& Zurek (2002). With Virgo E we refer to other ellipticals in the Virgo clusters.

\begin{tabular}{lllll}
\hline \hline Galaxy & Hubble type & novae $\left(\mathrm{yr}^{-1}\right)$ & $L_{K}\left(10^{10} L_{\odot, K}\right)$ & $\operatorname{LSNR}_{K}\left(\mathrm{yr}^{-1}\left(10^{10} L_{\odot, K}\right)^{-1}\right)$ \\
\hline M 31 & Sb I-II & $29 \pm 4$ & $18.5 \pm 2.4$ & $1.56 \pm 0.30$ \\
M 33 & Sc II-III & $4.6 \pm 0.9$ & $1.17 \pm 0.15$ & $3.93 \pm 0.93$ \\
M 51 & Sbc I-II & $18 \pm 7$ & $16.6 \pm 3.4$ & $1.09 \pm 0.47$ \\
M 81 & Sb I-II & $24 \pm 8$ & $13.3 \pm 2.7$ & $1.80 \pm 0.71$ \\
M 87 & E0 & $91 \pm 34$ & $39.8 \pm 8.2$ & $2.30 \pm 0.99$ \\
M 87* & & 200 to 300 & & \\
M 101 & Sc I & $12.0 \pm 4.0$ & $12.4 \pm 2.6$ & $0.97 \pm 0.38$ \\
NGC 5128 & S0(Pec. $)$ & $28 \pm 7$ & $7.94 \pm 1.10$ & $3.52 \pm 1.01$ \\
LMC & SBm III & $2.5 \pm 0.5$ & $0.46 \pm 0.06$ & $5.42 \pm 1.30$ \\
SMC & Im IV-V & $0.7 \pm 0.2$ & $0.14 \pm 0.02$ & $2.19 \pm 1.49$ \\
Virgo & E & $160 \pm 57$ & $49.7 \pm 10.5$ & $3.22 \pm 1.33$ \\
NGC 1316 & S0(Pec. $)$ & 90 to 180 & $?$ & $?$ \\
\hline
\end{tabular}

(e.g. Livio 2000). Whether these systems can give rise to novae or supernovae depends crucially on the mass of the underlying white dwarf and mass accretion rate (see Nomoto et al. 1984). Therefore, when trying to predict nova rates in galaxies it is obvious to compute and try to reproduce the observed SNIa rates. The observed type Ia rates in galaxies of different morphological type are shown in Table 2.

While the rates of novae are expressed in $\mathrm{yr}^{-1}$ or in $L S N R_{K}$, the rates of SNIa are expressed in $S N u_{B}$ (SN $\left.(100 \mathrm{yr})^{-1} 10^{-10} L_{B_{\odot}}\right)$ and $\mathrm{SNu}_{K},\left(\mathrm{SN}(100 \mathrm{yr})^{-1} 10^{-10} L_{K_{\odot}}\right)$. The paper is organized as follows: in Sect. 2 we describe how to compute the nova and $\mathrm{SN}$ rates in galaxies and compare the results with observations and finally, in Sect. 3, we draw some conclusions.

\section{Modelling the nova and supernova rates}

\subsection{The Milky Way}

The model for the Galaxy assumes two main infall episodes for the formation of the halo-thick disk, and the thin-disk, respectively. The timescale for the formation of the thin-disk is much longer than that of the halo, implying that the infalling gas forming the thin-disk comes not only from the halo but mainly from the intergalactic medium. The timescale for the formation of the thin disk is assumed to be a function of the galactocentric distance, leading to an inside-out picture for the Galaxy disk build-up, according to the original suggestion of Matteucci \& François (1989). The two-infall model differs from other models in the literature mainly in two aspects: it considers an almost independent evolution between the halo and thin-disk components (see also Pagel \& Tautvaisiene 1995), and it assumes a threshold in the star formation process, (see Kennicutt 1989, 1998; van der Hulst et al. 1993; Martin \& Kennicutt 2001). The model well reproduces the majority of observational constraints about the abundances of heavy elements both locally and in the whole disk.
Table 2. SNIa rate in $S N u_{B}$ from the combined search sample of Cappellaro et al. (1999) and SNIa rate in $S N u_{K}$ (Della Valle \& Livio 1994) $S N u_{B}$ and $S N u_{K}$ are in units of $S N(100 \mathrm{yr})^{-1} 10^{-10} L_{B_{\odot}}$ and $S N$ $(100 \mathrm{yr})^{-1} 10^{-10} L_{K_{\odot}}$, respectively, for $h=0.75$.

\begin{tabular}{lll}
\hline \hline Hubble type & SNeIa $\left(S N u_{B}\right)$ & SNeIa $\left(S N u_{K}\right)$ \\
\hline E-S0 & $0.18 \pm 0.06$ & $0.030 \pm 0.01$ \\
Sa-Sb & $0.18 \pm 0.07$ & $0.045 \pm 0.015$ \\
Sc-Sm & $0.40 \pm 0.08$ & $0.140 \pm 0.03$ \\
\hline
\end{tabular}

The star formation rate (SFR) adopted here has the same formulation as in Chiappini et al. (1997):

$\psi(R, t)=v(t)\left(\frac{\Sigma(R, t)}{\Sigma\left(R_{\odot}, t\right)}\right)^{2(k-1)}\left(\frac{\Sigma\left(R, t_{\mathrm{Gal}}\right)}{\Sigma(R, t)}\right)^{k-1} G_{\mathrm{gas}}^{k}(R, t)$

where $v(t)$ is the efficiency of the star formation process, $\Sigma(R, t)$ is the total surface mass density at a given radius $R$ and given time $t, \Sigma\left(R_{\odot}, t\right)$ is the total surface mass density at the solar position, $G_{\text {gas }}(R, t)$ is the surface gas density normalized to the present time total surface mass density in the disk $\Sigma_{D}\left(R, t_{\mathrm{Gal}}\right)$, $t_{\text {Gal }}=13 \mathrm{Gyr}$ is the age of the Galaxy, $R_{\odot}=8 \mathrm{kpc}$ is the solar galactocentric distance (see Reid 1993).

The gas surface density exponent, $k$, is set equal to 1.5 , in order to ensure a good fit to the observational constraints in the solar vicinity. This value is also in agreement with the observational results of Kennicutt (1998), and with N-body simulation results by Gerritsen \& Icke (1997). The star formation efficiency is set to $v=2 \mathrm{Gyr}^{-1}$ for the Galactic halo, whereas it is $v=1 \mathrm{Gyr}^{-1}$ for the disk; this is to ensure the best fit to the observational features in the solar vicinity. The star formation rate becomes zero when the gas surface density drops below a certain critical threshold. We adopt such a threshold density to be $\left(\sigma_{\text {gas }}\right)_{\mathrm{th}} \approx 4 M_{\odot} \mathrm{pc}^{-2}$ for the Galactic halo, and $\left(\sigma_{\text {gas }}\right)_{\text {th }} \approx 7 M_{\odot} \mathrm{pc}^{-2}$ for the disk (see Chiappini et al. 2001). The assumption of such threshold densities naturally produces the existence of a hiatus in the SFR between the halo-thick disk phase and the thin-disk phase. This discontinuity in the SFR is observed in the $[\mathrm{Fe} / \mathrm{O}]$ vs. $[\mathrm{O} / \mathrm{H}]$ (Gratton et al. 2000) and in 
Table 3. Results for the model for the Galaxy, IMF Scalo (1986), $\alpha=$ $0.01, A=0.05, t_{\mathrm{Gal}}=13 \mathrm{Gyr}$.

\begin{tabular}{llll}
\hline \hline nova $\left(\mathrm{yr}^{-1}\right)$ & nova $\left(L S N R_{K}\right)$ & SNeIa $\left(100 \mathrm{yr}^{-1}\right)$ & SNeIa $(\mathrm{SNu})$ \\
\hline 25 & 2.1 & 0.3 & 0.16 \\
\hline
\end{tabular}

the $[\mathrm{Fe} / \mathrm{Mg}]$ vs. $[\mathrm{Mg} / \mathrm{H}]$ (Fuhrmann 1998) plots. The IMF is that of Scalo (1986), assumed to be constant in time and space. We compute the nova systems formation rate $\left(R_{\text {novae }}(t)\right)$ at the time $t$ as a fraction of the formation rate of white dwarfs at a previous time $t-\Delta t$ (see D'Antona \& Matteucci 1991):

$R_{\text {novae }}(t)=\alpha \int_{0.8}^{8.0} \psi\left(t-\tau_{m}-\Delta t\right) \phi(m) \mathrm{d} m$.

Here $\Delta t$ is a delay time whose value has to be fixed to guarantee the cooling of the white dwarf (WD) to a level that ensures a strong enough nova outburst. We assume a distribution of $\Delta t$ varying from 1 to $5 \mathrm{Gyr}$. It is worth noting that assuming a constant average delay of $\Delta t=2 \mathrm{Gyr}$, as assumed in previous papers (e.g. Romano et al. 1999, 2001), produces negligible differences in the final results.

It is assumed that all stars with initial masses in the range $0.8-8 M_{\odot}$ end their lives as WDs. $\psi(\mathrm{t})$ is the SFR as defined in Eq. (1), $\tau_{m}$ is the lifetime of the star of mass $m$ and $\phi(m)$ is the IMF

The rate of nova eruptions is related to the WD formation rate by:

$R_{\text {outbursts }}(t)=\alpha R_{\mathrm{WDs}}(t) n$,

where $\alpha R_{\mathrm{WDs}}=R_{\text {novae }}(t)$ is the formation rate of WDs in binary systems, as defined in Eq. (2), which will give rise to nova eruptions and $n=10^{4}$ is the average number of nova outbursts for a typical nova system (Ford 1978; Bath \& Shaviv 1978; Shara et al. 1986). This number, which is just an average value, is inversely proportional to the recurrence time. The parameter $\alpha$, which here is a constant in space and time, can be fixed by reproducing the rate of nova outbursts in our Galaxy at the present time. Unfortunately, estimates of this quantity in the current literature show a large spread. Predictions based on scalings from extragalactic nova surveys suggest low values, whereas estimates based on extrapolations of Galactic nova observations give the highest rates. We consider for the present time observed rate of nova outbursts in the Galaxy $R_{\text {outbursts }}\left(t_{\mathrm{Gal}}\right) \approx 25 \mathrm{yr}^{-1}$, as in Romano et al. (1999), for the following reasons. Given the fact that observations of novae in nearby galaxies would avoid, or at least minimize, most of the problems encountered by Galactic observations, such as interstellar extinction in the Galactic disk, Shafter (1997) shows that the nova rate based on Galactic observations can be made consistent with the rate predicted from the extragalactic data (Della Valle et al. 1994), particularly if the Galaxy has a strong bar oriented in the direction of the Sun. In this latter, most favourable case, the suggested value is $\approx 20 \mathrm{yr}^{-1}$, otherwise, if the bar is weak or misaligned, the global rate can be $\approx 30 \mathrm{yr}^{-1}$.

Our first choice is to fix $\alpha=0.01$ (coupled with $n=10^{4}$ ) so to reproduce the present observed nova rate of $25 \mathrm{yr}^{-1}$ and to use the same $\alpha$ in the models for elliptical and irregular galaxies. This value of $\alpha$ is the same as in previous papers (e.g. Romano et al. 2001; Matteucci et al. 1999).

Finally, the SNeIa rate has been computed following Greggio \& Renzini (1983) (see also Matteucci \& Greggio 1986) and is expressed as:

$R_{\mathrm{SNeIa}}=A \int_{M_{B_{m}}}^{M_{B_{M}}} \phi\left(M_{B}\right) \int_{\mu_{m}}^{0.5} f(\mu) \psi\left(t-\tau_{M_{2}}\right) \mathrm{d} \mu \mathrm{d} M_{B}$,

where $M_{2}$ is the mass of the secondary, $M_{B}$ is the total mass of the binary system, $\mu=M_{2} / M_{B}, \mu_{m}=\max \left\{M_{2}(t) / M_{B},\left(M_{B}-\right.\right.$ $\left.\left.0.5 M_{B_{M}}\right) / M_{B}\right\}, M_{B_{m}}=3 M_{\odot}, M_{B_{M}}=16 M_{\odot} . \phi\left(M_{B}\right)$ is the initial mass function for the total mass of the binary system, $f(\mu)$ is the distribution function for the mass fraction of the secondary, $f(\mu)=2^{1+\gamma}(1+\gamma) \mu^{\gamma}$, with $\gamma=2 ; A=0.05$ is the fraction of systems, with total mass in the appropriate range, which give rise to SNIa events. This quantity is fixed by reproducing the observed SNe Ia rate at the present epoch (see also Madau et al. 1998), and it provides excellent fits to the abundances and abundance ratios in the Galaxy.

The results for the Galaxy are shown in Table 3 and Fig. 1. In Table 3 we report the predicted nova and SN Ia rate for the Milky Way, adopting an age of $13 \mathrm{Gyr}$, according to a cosmology with $\Omega_{m}=0.3, \Omega_{\Lambda}=0.7, h=0.7$ and $z_{f}=10$. We computed the $K$ - and $B$-band luminosity of the Milky Way by means of the spectro-photometric model of Jimenez et al. (1999): we find, for the present time, luminosities in very good agreement with the estimated ones. The estimated Galactic blue luminosity is $L_{B_{\mathrm{Gal}}} \sim 2 \times 10^{10} L_{\odot}$ (see van den Bergh 1988) and the $K$-luminosity is $L_{K_{\mathrm{GAL}}} \sim 12 \times 10^{10} L_{\odot}$ (see Shafter el al. 2000). In Fig. 1 we show the behaviour of the star formation rate in the framework of the two-infall model, where the gap between the formation of halo-thick disk and thin-disk is evident, as well as the behaviour of the nova and SN Ia rates. These latter show only a small discontinuity in correspondance with the star formation gap and the reason is that they both depend upon the past star formation. The nova rate increases continuously up to the present time due to the long delay assumed for these systems (the lifetime of the WD plus the $\Delta t$ ), whereas the SNIa rate reaches a maximum and then it stays flat afterwards.

\subsection{Ellipticals}

In the models for ellipticals, the galaxies can be considered initially as an homogeneous sphere of gas with luminous masses in the range $10^{9}-10^{12} M_{\odot}$. A single zone interstellar medium with instantaneous mixing of gas is assumed throughout.

The adopted model parameters are shown in Tables 4 and 5, where we show the initial luminous masses for the model galaxies, the assumed star formation efficiency parameter, the effective radius, the predicted time for the occurrence of a galactic wind $t_{\mathrm{GW}}$ and the predicted $K$ - and $B$-luminosities. The parameter $A$ is varied according to the assumed IMF in order to reproduce the present time observed type Ia SN rate (Matteucci $\&$ Gibson 1995). On the other hand, we kept the parameter $\alpha$ to be the same as in the Milky Way. The model is described in 
Table 4. Model I: $x=1.35, \alpha=0.01, A=0.18, t_{\mathrm{gal}}=13 \mathrm{Gyr}$.

\begin{tabular}{llllll}
\hline \hline$M_{\mathrm{lum}}\left(M_{\odot}\right)$ & $v\left(\mathrm{Gyr}^{-1}\right)$ & $R_{\mathrm{eff}}(\mathrm{kpc})$ & $t_{\mathrm{GW}}(\mathrm{Gyr})$ & $L_{K}\left(10^{10} L_{K_{\odot}}\right)$ & $L_{B}\left(10^{10} L_{B_{\odot}}\right)$ \\
\hline $10^{9}$ & 2. & 0.5 & 1.4 & 0.038 & 0.0091 \\
$10^{10}$ & 5. & 1.0 & 1.3 & 0.40 & 0.093 \\
$10^{11}$ & 11. & 3.0 & 0.6 & 5.9 & 0.70 \\
$4 \times 10^{11}$ & 15. & 6.0 & 0.4 & 17.0 & 3.9 \\
$6 \times 10^{11}$ & 16.5 & 7.0 & 0.4 & 25.0 & 5.9 \\
$10^{12}$ & 20. & 10.0 & 0.4 & 45.0 & 9.6 \\
\hline
\end{tabular}

Table 5. Model II: $x=0.95, \alpha=0.01, A=0.05$, $t_{\mathrm{gal}}=13 \mathrm{Gyr}$.

\begin{tabular}{llllll}
\hline \hline$M_{\mathrm{lum}}\left(M_{\odot}\right)$ & $v\left(\mathrm{Gyr}^{-1}\right)$ & $R_{\mathrm{eff}}(\mathrm{kpc})$ & $t_{\mathrm{GW}}(\mathrm{Gyr})$ & $L_{K}\left(10^{10} L_{K_{\odot}}\right)$ & $L_{B}\left(10^{10} L_{B_{\odot}}\right)$ \\
\hline $10^{9}$ & 2. & 0.5 & 1.4 & 0.034 & 0.0044 \\
$10^{10}$ & 5. & 1.0 & 0.9 & 0.42 & 0.049 \\
$10^{11}$ & 11. & 3.0 & 0.6 & 4.3 & 0.53 \\
$4 \times 10^{11}$ & 15. & 6.0 & 0.4 & 19.0 & 2.3 \\
$6 \times 10^{11}$ & 16.5 & 7.0 & 0.4 & 37.0 & 3.2 \\
$10^{12}$ & 20. & 10.0 & 0.3 & 47.7 & 6.2 \\
\hline
\end{tabular}

Matteucci et al. (1998) where we address the reader for more details.

The star formation rate is given by:

$\psi(t)=v \rho_{\mathrm{gas}}(t) / \rho(0)$,

i.e. normalized to the initial total volume density. $\psi(t)$ is assumed to drop to 0 at the onset of the galactic wind. The quantity $v$ is expressed in units of $\mathrm{Gyr}^{-1}$ and represents the efficiency of star formation, namely the inverse of the time scale of star formation. The star formation is assumed to stop after the development of a galactic wind occurring before than $1 \mathrm{Gyr}$, from the beginning of star formation, for all the galaxies listed above. Therefore, the star formation rate in these galaxies can be considered as a strong burst which does not last more than $1 \mathrm{Gyr}$ and is shorter in more massive systems. This is obtained by assuming that the star formation efficiency increases with galactic mass thus producing an "inverse wind" effect, as described in Matteucci (1994), where the galactic wind occurs before in more massive than in less massive ellipticals. As a consequence of this, the star formation period is longer in smaller systems thus allowing the SNe Ia to substantially pollute the ISM. This effect can explain the observed increase of the $[\alpha / \mathrm{Fe}]$ ratio with galactic mass (Worthey et al. 1992; Matteucci 1994), which is not obtained in a classic wind scenario, where the more massive objects form stars for a longer time (Larson 1974). The galactic wind develops as a consequence of the energy transfer from $\mathrm{SNe}$ into the ISM. In fact, when the thermal energy of the gas becomes larger than the binding energy of the gas, the wind starts (Arimoto \& Yoshii 1987; Matteucci \& Tornambe 1987; Matteucci 1992, 1994; Pipino et al. 2002). In order to compute the binding energy of the gas some assumptions have to be made about the galactic potential well. In particular, it is assumed that all ellipticals possess heavy but diffuse dark matter halos; a ratio between the half-light radius and the radius of the dark matter core $R_{\text {luminous }} / R_{\text {dark }}=0.10$ and a ratio dark to luminous mass of 10 are assumed.

The $B$ and $K$ luminosities for the ellipticals of different masses are computed by means of the spectro-photometric model of Jimenez et al. (1999) and are used to compute the SN Ia rate in $\mathrm{SNu}$ and the nova rate per unit of $L_{K}$, respectively.

The main differences between the model for the Galaxy, and the model for an elliptical galaxy concern the different SFR, which is much stronger in the earliest stages, and then is set to zero after the galactic wind in the case of ellipticals. It is worth noting that galactic winds in the case of elliptical galaxies seem necessary to explain their lack of gas and the chemical enrichment of the ICM, whereas the Galactic model does not take into account the occurrence of a strong wind, mainly because of the strong gravitational potential well associated with our Galaxy, and the presence of the gas in the Galactic disk.

We adopt two different initial mass functions (IMFs): the Salpeter $\left(x_{\mathrm{IMF}}=1.35\right)$ one and the Arimoto \& Yoshii (1987) $\left(x_{\mathrm{IMF}}=0.95\right)$ one. In fact, successful models of chemical evolution of ellipticals have shown that the Scalo IMF is not suitable for these galaxies (e.g. Matteucci \& Gibson 1995).

Table 6 shows the results of the model I (Salpeter IMF), whereas Table 7 shows the results of the model II $(x=$ $0.95 \mathrm{IMF}$ ). These models have been computed by assuming the same $\alpha$ and the same $n$ as for the Milky Way.

In particular, in the first column we report the initial galactic luminous mass, in the second column the nova rate in units of $\mathrm{yr}^{-1}$, in Col. 3 the luminosity specific nova rate, in Col. 4 the SN Ia rate in units of $(100 \mathrm{yr})^{-1}$ and in Cols. 5 and 6 the SN Ia rate in units of $S N u_{B}$ and $S N u_{K}$, respectively. The predicted nova rates are quite large for massive ellipticals ranging between 300 and 1000 nova $\mathrm{yr}^{-1}$ which is about a factor 3-10 larger than it is derived from observations. If these values were realistic, the discrepancy could be partially due to an 
Table 6. Results for model I: nova and SN Ia rates.

\begin{tabular}{llllll}
\hline \hline$M_{\text {lum }}\left(M_{\odot}\right)$ & novae $\left(\mathrm{yr}^{-1}\right)$ & novae $\left(L S N R_{K}\right)$ & SNe Ia $\left(100 \mathrm{yr}^{-1}\right)$ & SNe Ia $\left(S N u_{B}\right)$ & SNe Ia $\left(S N u_{K}\right)$ \\
\hline $10^{9}$ & 0.6 & 15.8 & 0.0026 & 0.28 & 0.07 \\
$10^{10}$ & 8 & 20.0 & 0.027 & 0.29 & 0.08 \\
$10^{11}$ & 97 & 16.4 & 0.19 & 0.27 & 0.03 \\
$4 \times 10^{11}$ & 409 & 24.0 & 0.54 & 0.14 & 0.03 \\
$6 \times 10^{11}$ & 635 & 25.4 & 0.78 & 0.13 & 0.03 \\
\hline
\end{tabular}

Table 7. Results for model II: nova and SN Ia rates.

\begin{tabular}{llllll}
\hline \hline$M_{\text {lum }}\left(M_{\odot}\right)$ & novae $\left(\mathrm{yr}^{-1}\right)$ & novae $\left(L S N R_{K}\right)$ & SNe Ia $\left(100 \mathrm{yr}^{-1}\right)$ & SNe Ia $\left(S N u_{B}\right)$ & SNe Ia $\left(S N u_{K}\right)$ \\
\hline $10^{9}$ & 0.6 & 17.6 & 0.0012 & 0.27 & 0.03 \\
$10^{10}$ & 7 & 16.7 & 0.011 & 0.22 & 0.03 \\
$10^{11}$ & 89 & 20.7 & 0.11 & 0.21 & 0.02 \\
$4 \times 10^{11}$ & 374 & 19.7 & 0.35 & 0.15 & 0.02 \\
$6 \times 10^{11}$ & 585 & 15.8 & 0.51 & 0.16 & 0.014 \\
$10^{12}$ & 1013 & 21.2 & 0.40 & 0.06 & 0.001 \\
\hline
\end{tabular}

observational bias affecting the ground based nova surveys due to poor spatial resolution and bright limiting magnitude. An indication in this direction comes from the nova rate of M 87 recently provided by Shara \& Zurek (2002) on the basis of HST archive images, which is a factor 2-3 larger than previous ground-based estimates (see Table 1). Another possibility is that in early type galaxies the recurrence time between two consecutive nova explosions is considerably longer than in late spirals as a consequence of the different stellar populations from which novae originate. This suggestion is supported both by observational and theoretical grounds. From one side, Duerbeck (1990) and Della Valle et al. $(1992,1994)$ have demonstrated, on the basis of galactic and extragalactic nova observations, the existence of two populations of novae: fast and bright novae belonging to "disk" stellar population and slow and faint novae which originate from a "bulge" stellar population. In particular, the latter authors (see also Della Valle \& Livio 1998) suggested that bulge novae could originate from relatively light WDs, likely in the range of masses of $\left\langle M_{\mathrm{WD}}\right\rangle \leq 0.9 M_{\odot}$ while novae in the disk arise from massive WDs $\left(\left\langle M_{\mathrm{WD}}\right\rangle \geq 1 M_{\odot}\right)$. From the other side, Truran (1990, see also Ritter et al. 1991) has found that the mass of the WD and the recurrence time between the outbursts are inversely proportional. In order to match the "theoretical" with "empirical" rates, one needs to lower either $\alpha$ or $n$ or both. For example, for M 87 the match between predicted and observed rates can be achieved by increasing the recurrence time between two consecutive outbursts, to $T_{\mathrm{r}} \sim 3-1 \times 10^{5} \mathrm{yr}$. This is about 10-3 times larger than assumed for novae in the "disk" of the Galaxy. In this way, both the predictions of models I and II, for a luminous mass in the range $4-6 \times 10^{11} M_{\odot}$ are in very good agreement with the preliminary new estimate from Shara \& Zurek (2002) of the nova rate for M 87. In fact, M 87 can be modeled both as an elliptical galaxy with luminous mass $\sim 4 \times 10^{11} M_{\odot}$ and $R_{\text {eff }} \sim 6 \mathrm{kpc}$ or with luminous mass $\sim 6 \times 10^{11} M_{\odot}$ and $R_{\text {eff }} \sim 7 \mathrm{kpc}$ (Cohen \& Ryzhov 1997). The predictions of these two models do not differ much and both of them can well represent M 87. Another possibility is to adopt a fraction of binary systems giving rise to novae smaller than 0.01. Unfortunately, if from one side we can justify this value for the Milky Way because this $\alpha$ gives both the observed present time Galactic rate and the correct yields of lithium and CNO isotopes (see Romano \& Matteucci 2003), on the other side, we completely lack this information for ellipticals.

Finally, a comparison of Tables 6 and 7 with Table 2 shows a very good agreement between the observed and predicted type Ia SN rates both in units of $S N u_{B}$ and $S N u_{K}$.

In Figs. 2 and 3 we show the predicted star formation, nova and SNIa rates as functions of the cosmic time for ellipticals of different luminous masses and for the two IMFs.

Figure 4 shows the nova rate in the ellipticals as a function of the mass of the galaxy. From this figure one can infer the expected nova rate as a function of luminous galactic mass.

\subsection{Magellanic irregulars}

As an example of Magellanic irregular galaxies and late-type spirals we computed a model to fit the LMC. In particular, we assumed an history of star formation with two main bursts occurring during the first $3 \mathrm{Gyr}$ of the galactic life and during the last 2 Gyr, respectively, coupled with a lower star formation rate in between the two, as shown in Fig. 5. This star formation regime for the LMC has been suggested by Calura et al. (2003) and it can reproduce the observed [O/Fe] versus $[\mathrm{Fe} / \mathrm{H}]$. The star formation rate is like in Eq. (5) with an efficiency of star formation $v=0.1 \mathrm{Gyr}^{-1}$ in each starburst and of $v=0.01 \mathrm{Gyr}^{-1}$ in the interburst period. The IMF is the Salpeter one and the parameters $\alpha=0.01$ and $A=0.18$. The history of star formation has been chosen to reproduce $[\mathrm{O} / \mathrm{Fe}]$ vs. $[\mathrm{Fe} / \mathrm{H}]$ in the LMC (see Fig. 5 and Calura et al. 2003). We considered a luminous mass of $\sim 10^{10} M_{\odot}$ which is suitable for the LMC (e.g. Russell \& Dopita 1992). We adopted the same values of $\alpha$ and $n$ as for the Milky Way and found that the predicted present time nova rate is in excellent agreement with the observed one and is $\sim 1.7$ nova $\mathrm{yr}^{-1}$. The predicted $B$ and $K$ luminosities are 

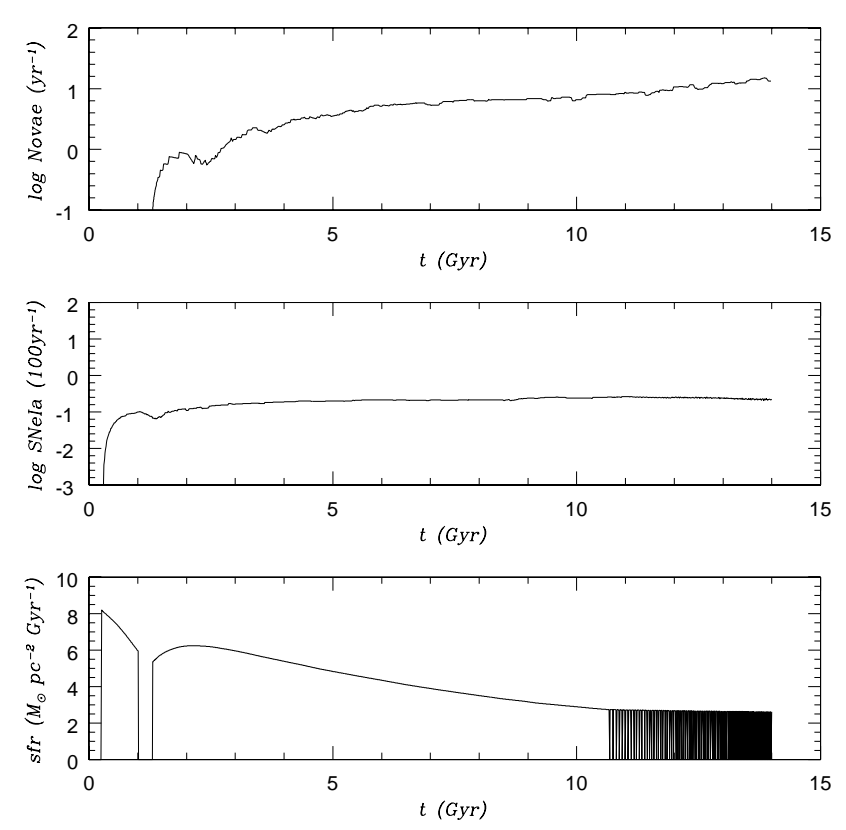

Fig. 1. Nova rate, SNeIa rate and SFR predictions for the "two-infall" model for the Galaxy. Note the oscillatory behaviour of the SFR during the last 2 or $3 \mathrm{Gyr}$.
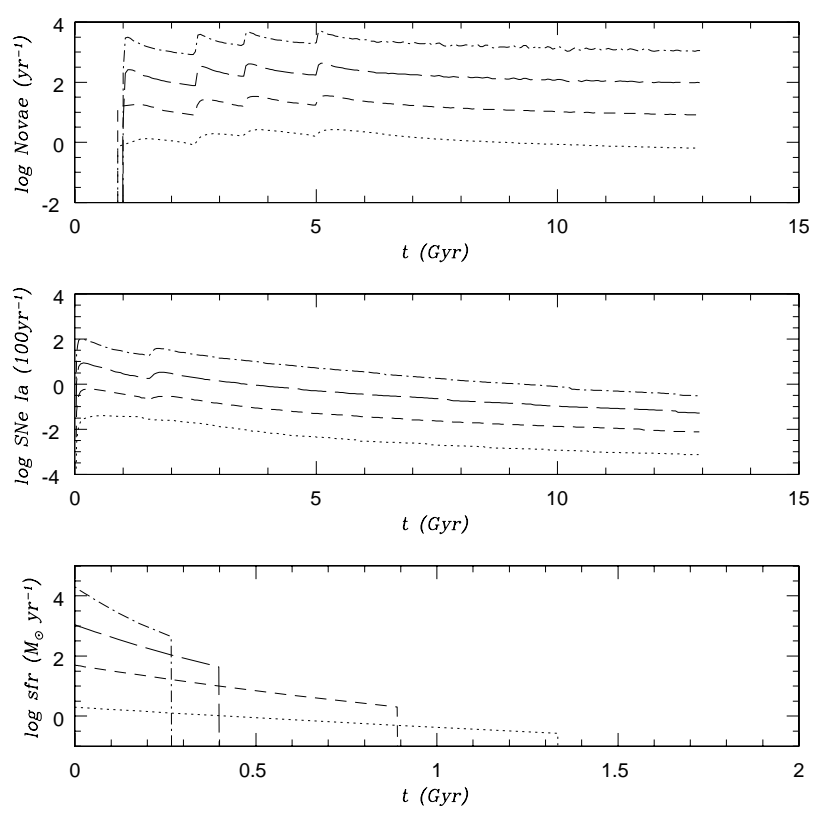

Fig. 2. Ellipticals: star formation rate, SNeIa rate and nova rate predictions for model I. Dotted, $10^{9} M_{\odot}$; short-dashed, $10^{10} M_{\odot}$; longdashed, $10^{11} M_{\odot}$; dotted-short-dashed, $10^{12} M_{\odot}$.

$L_{B}=2 \times 10^{9} L_{\odot}$ and $L_{K}=0.27 \times 10^{10} L_{\odot}$, respectively. As a consequence, we predict a nova rate in units of $L N S R_{K}$ of $\sim 6$, in agreement with the data of Table 1 . The predicted type Ia SN rate is $\sim 0.31 \mathrm{SNu}$, also in very good agreement with the data of Table 2 .

\section{Discussion and conclusions}

In this paper we have computed the Galactic and extragalactic nova rates, in particular the nova rates expected in elliptical
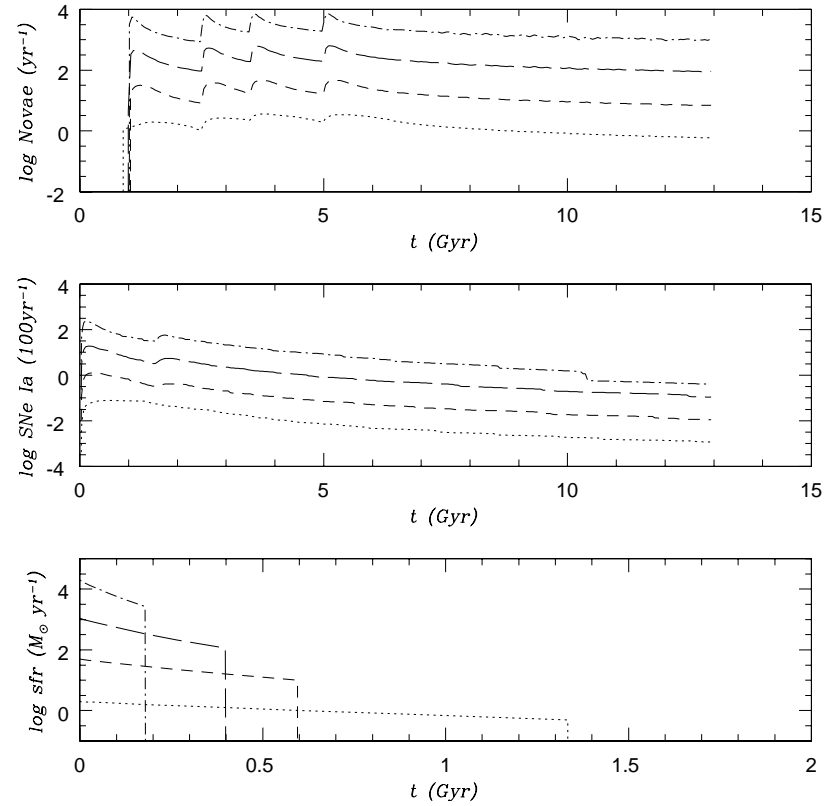

Fig. 3. Ellipticals: star formation rate, SNeIa rate and nova rate predictions for model II. Dotted, $10^{9} M_{\odot}$; short-dashed, $10^{10} M_{\odot}$; longdashed, $10^{11} M_{\odot}$; dotted-short-dashed, $10^{12} M_{\odot}$.

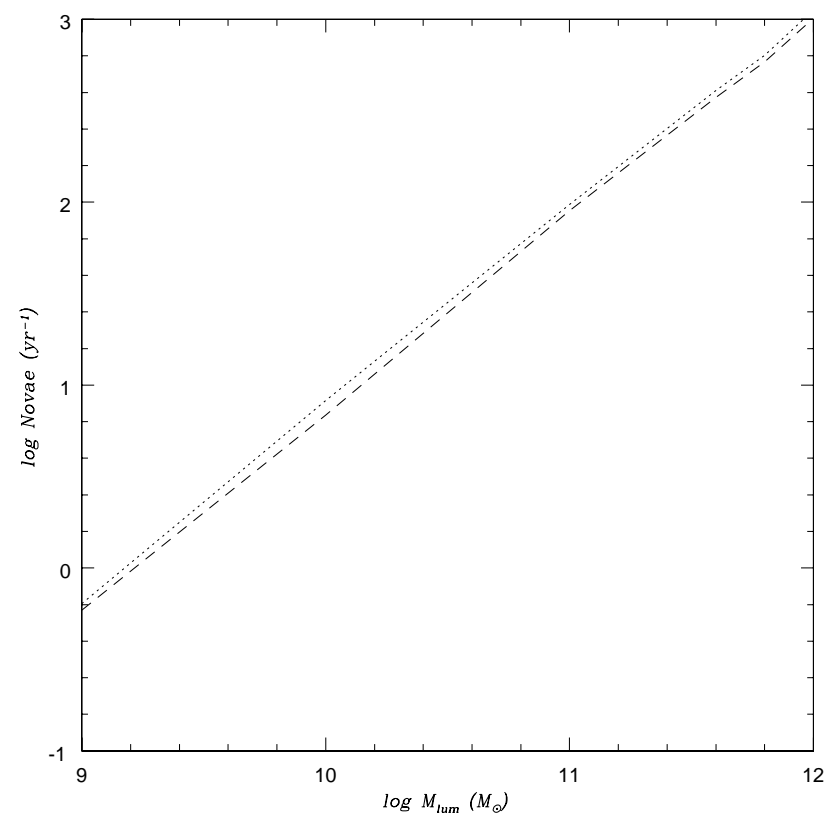

Fig. 4. Ellipticals: nova rate as a function of $M_{\text {lum }}$ of the galaxy: model I (dotted), model II (short-dashed).

galaxies and in Magellanic irregulars, typified by the LMC. To do that we have adopted detailed models of chemical and photometric evolution of the Milky Way, ellipticals and the LMC. These models can reproduce the majority of the observational constraints, including abundances, abundance ratios, luminosities and colors. We have computed also the type Ia $\mathrm{SN}$ rates since the novae and type Ia $\mathrm{SNe}$ are originating by $\mathrm{CV}$-like binary systems. The nova rate is calculated in a simple way but still depends on several unknown parameters such as the fraction of binary systems which are nova progenitors (parameter $\alpha$ ) and the total number of outbursts occurring during a 

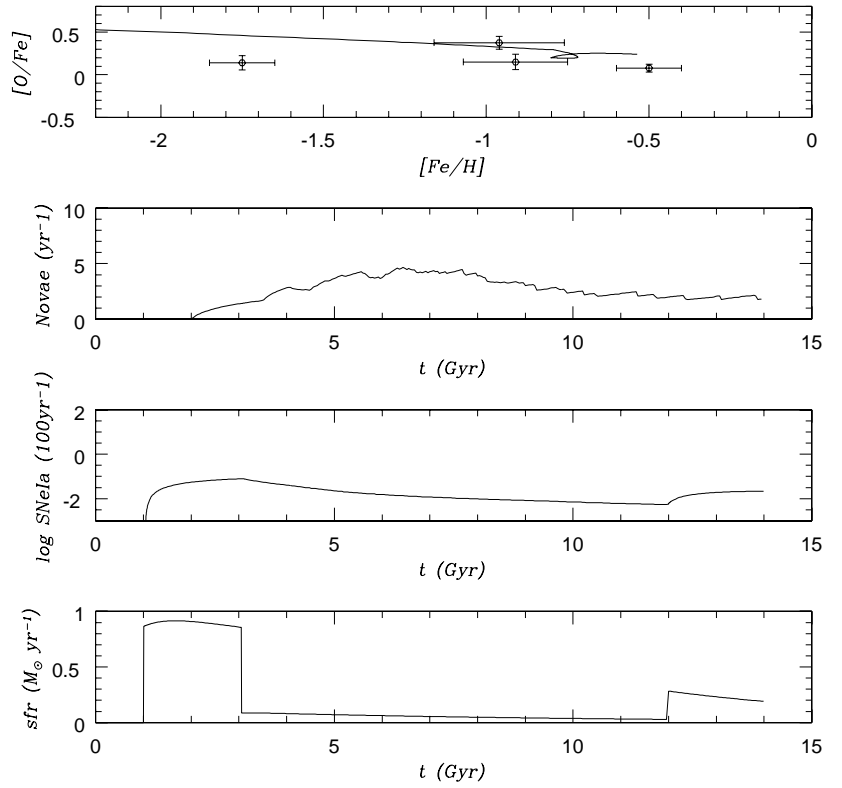

Fig. 5. Irregulars: star formation rate, SNeIa rate, nova rate and $[\mathrm{O} / \mathrm{Fe}]$ vs. $[\mathrm{Fe} / \mathrm{H}]$ predictions for the LMC. The data are from Hill et al. (2000).

nova lifetime (parameter $n$ ). For the sake of simplicity, we have assumed these two parameters to be the same in all galaxies whereas the star formation histories are different and tuned to reproduce the available observational constraints. For the Milky Way this particular choice of parameters, besides reproducing the observed nova rate, allows to predict ${ }^{7} \mathrm{Li},{ }^{15} \mathrm{~N},{ }^{13} \mathrm{C}$ and ${ }^{17} \mathrm{O}$, in good agreement with the observations (Romano $\&$ Matteucci 2003). On the other hand, for external galaxies we do not have other constraints related to novae except the nova rates. Therefore, this approach does not allow us to put strong constraints upon $\alpha$ and $n$ singularly but rather on the product $\alpha \cdot n$. Concerning the delay with which novae appear since the beginning of star formation, we find that an average delay of 2 Gyr can be assumed for all the studied galaxies.

Our main conclusions can be summarized as follows:

- We predict that the nova rates in ellipticals reach a maximum after $\sim 10^{9}$ years since the beginning of star formation and then they decrease after that. The same behaviour is shared by type Ia SNe although they reach a maximum after only a few $10^{8}$ years (see also Matteucci \& Recchi 2001). The delayed maximum in the nova rate relative to the SNIa rate is due to the longer time delay assumed for novae to appear relative to SNeIa (see Canal et al. 1996).

- Our results are suggesting that a model for ellipticals which assumes a strong burst of star formation occurring at early cosmic times can reproduce the majority of the observational constraints in ellipticals. The same model predicts that the nova rates are increasing with the galactic mass of ellipticals and for a galaxy like M 87 we predict a present time nova rate of $300-1000$ nova $\mathrm{yr}^{-1}$, when the values of $\alpha$ and $n$ are the same as for the Galaxy. A good agreement with the observations (100-300 nova $\mathrm{yr}^{-1}$ ) is obtained if we assume an average recurrence time between two consecutive nova outbursts of the order of $1-3 \times 10^{5} \mathrm{yr}$, which is a factor of 10 larger than the typical recurrence time for novae originating in the disk of the Milky Way. This fact is a consequence of the existence of two populations of novae, one associated with disk/spiral arms stellar population and the other with bulge/thick disk stellar population (Della Valle et al. 1994; Yungelson et al. 1997).

- The nova rate in ellipticals is rather independent from the adopted IMF, but it depends on the assumption that the efficiency of star formation is an increasing function of the luminous galactic mass (inverse wind model of Matteucci 1994). We do not predict any trend for the luminosity specific nova rate as a function of the galactic mass for these galaxies.

- We predict also a type Ia SN rate for ellipticals in very good agreement with the observed estimates and a slight decreasing trend for the SNIa rate with galactic blue and red luminosity.

- Finally, we confirm the existence of a trend for the luminosity specific nova rate, as derived from the observations (Della Valle et al. 1994), passing from a Magellanic irregular (spiral) like the LMC, $\sim 6$, to a Sb spiral galaxy like the Milky Way $\sim 2.1$. For a giant elliptical like M 87 we obtain a large range of uncertainty between 2.5 and 7.5 novae/yr per unit of $10^{10} \mathrm{~K}$ luminosity. The lower limit of the rate is very similar to that obtained from ground based nova survey whereas the upper limit is consistent with nova rates obtained from HST observations (Shara \& Zurek 2002). If true, this could indicate that the studies of novae in extragalactic systems from ground-based telescopes, can be largely affected by observational bias. As an alternative, we note that Livio et al. (2002) have suggested that M 87 could be a fast producer of novae, because of the presence of the jet which can enhance the accretion onto white dwarfs and therefore increase the nova rate.

Acknowledgements. We thank Donatella Romano for many useful suggestions and the referee H. W. Duerbeck for his careful reading of this manuscript.

\section{References}

Arimoto, N., \& Yoshii, Y. 1987, A\&A, 173, 23

Arp, H. C. 1956, AJ, 61, 15

Bath, G. T., \& Shaviv, G. 1978, MNRAS, 183, 515

Cappellaro, E., Evans, R., \& Turatto, M. 1999, A\&A, 351, 459

Calura, F., Matteucci, F., \& Vladilo, G. 2003, MNRAS, 340, 59

Canal, R., Ruiz-Lapuente, P., \& Burkert, A. 1996, ApJ, 456, L101

Chiappini, C., Matteucci, F., \& Gratton, R. 1997, ApJ, 477, 765

Chiappini, C., Matteucci, F., \& Romano, D. 2001, ApJ, 554, 1044

Cohen, J. G., \& Ryzhov, A. 1997, ApJ, 486, 230

D’Antona, F., \& Matteucci, F. 1991, A\&A, 248, 62

Della Valle, M. 2002, ed. M. Hernanz, \& J. Isern, AIP Conf. Proc., 637,443

Della Valle, M., \& Gilmozzi, M. 2002, Science, 296, 1275

Della Valle, M., Bianchini, A., Livio, M., \& Orio, M. 1992, A\&A, 266, 232

Della Valle, M., \& Livio, M. 1998, ApJ, 506, 818

Della Valle, M., \& Livio, M. 1994, A\&A, 286, 786 
Della Valle, M., Pasquini, L., Daou, D., \& Williams, R. E. 2002, A\&A, 390, 155

Della Valle, M., Rosino, L., Bianchini, A., \& Livio, M. 1994, A\&A, 287,403

Duerbeck, H. W. 1990, IAU Coll., 122, ed. A. Cassatella, \& R. Viotti (Berlin: Springer-Verlag), 34

Ford, H. C. 1978, ApJ, 219, 595

Furhmann, K. 1998, A\&A, 338, 161

Gehrz, R. D., Truran, J. W., Williams, R. E., \& Starrfield, S. 1998, PASP, 110,3

Gerritsen, J. P. E., \& Icke, V. 1997, A\&A, 325, 972

Gomez-Gomar, J., Hernanz, M., Jose, J., \& Isern, J. 1998, MNRAS, 296, 913

Gratton, R., Carretta, E., Matteucci, F., \& Sneden, C. 2000, A\&A, 358, 671

Greggio, L., \& Renzini, A. 1983, A\&A, 118, 217

Jimenez, R., Padoan, P., Matteucci, F., \& Heavens, A. F. 1998, MNRAS, 299, 123

Jose, J., \& Hernanz, M. 1998, ApJ, 494, 680

Kennicutt, R. C. Jr. 1989, ApJ, 344, 685

Kennicutt, R. C. Jr. 1998, ApJ, 498, 541

Hill, V., François, P., Spite, M., Primas, F., \& Spite, F. 2000, A\&A, 364, L19

Hubble, E. P. 1929, ApJ, 69, 103

Larson, R. B. 1974, MNRAS, 169, 229

Livio, M. 2000, in Type Ia SNe, Theory and Cosmology, ed. J. C. Niemayer, \& J. W. Truran (Cambridge Univ. Press), 33

Madau, P., Della Valle, M., \& Panagia, N. 1998, MNRAS, 297, L17

Martin, C. L., \& Kennicutt, R. C. Jr. 2001, ApJ, 555, 301

Matteucci, F., \& Francois, P. 1989, MNRAS, 239, 885

Matteucci, F., \& Greggio, L. 1986, A\&A, 154, 279

Matteucci, F., \& Tornambe, A. 1987, A\&A, 185, 51

Matteucci, F. 1992, ApJ, 397, 32

Matteucci, F. 1994, A\&A, 288, 57

Matteucci, F., \& Gibson, B. K. 1995, A\&A, 304, 11
Matteucci, F., Ponzone, R., \& Gibson, B. K. 1998, A\&A, 335, 855

Matteucci, F., \& Recchi, S. 2001, ApJ, 558, 351

Matteucci, F., Romano, D., \& Molaro, P. 1999, A\&A, 341, 458

Nomoto, K., Thielemann, F.-K., \& Yokoi, K. 1984, ApJ, 286, 644

Pagel, B. E. J., \& Tautvaisiene, G. 1995, MNRAS, 276, 505

Pipino, A., Matteucci, F., Borgani, S., \& Biviano, A. 2002, New Astron., 7, 227

Pritchet, C. J., \& van den Bergh, S. 1987, ApJ, 318, 507

Reid, M. J. 1993, ARA\&A, 31, 345

Ritter, H., Politano, M., Livio, M., \& Webbink, R. 1991, ApJ, 376, 177

Romano, D., Matteucci, F., Molaro, P., \& Bonifacio, P. 1999, A\&A, 352, 117

Romano, D., Matteucci, F., Ventura, P., \& D’Antona, F. 2001, A\&A, 374,646

Romano, D., \& Matteucci, F. 2003, MNRAS, in press

Rosino, L. 1964, Ann. Astrophys., 27, 497

Russell, S. C., \& Dopita, M. A. 1992, ApJ, 384, 508

Scalo, J. M. 1986, FCPh, 11, 1

Shafter, A. W. 1997, ApJ, 487, 226

Shafter, A. W., Ciardullo, R., \& Pritchet, C. J. 2000, ApJ 530, 193

Shara, M. M., Livio, M., Moffat, A. F. J., \& Orio, M. 1986, ApJ, 311, 163

Shara, M. M. 2000, New Astron. Rev., 44, 87

Shara, M. M., \& Zurek, D. R. 2002, in Classical Nova Explosions, ed. M. Hernanz, \& J. Josè, AIP Conf. Proc., 457

Shara, M. M., Sandage, A., \& Zurek, D. R. 1999, PASP, 111, 1367

Truran, J. W. 1990, IAU Coll., 122, ed. A. Cassatella, \& R. Viotti (Berlin: Springer-Verlag), 373

van den Bergh, S. 1988, ComAp, 12, 131

van der Hulst, J. M., Skillman, E. D., Smith, T., et al. 1993, AJ, 106, 548

Worthey, G., Faber, S. M., \& Gonzalez, J. J. 1992, ApJ, 398, 69

Yungelson, L., Livio, M., \& Tutukov, A. 1997, ApJ, 481, 127 